\title{
Sir George Mackenzie of Rosehaugh, Procureur du Roi, défenseur de la sorcière Maevia en 1672
}

\section{Marie-Claude Tucker}

\section{(2) OpenEdition}

1 Journals

\section{Édition électronique}

URL : http://journals.openedition.org/etudesecossaises/3469

DOI : 10.4000/etudesecossaises.3469

ISSN : 1969-6337

\section{Éditeur}

UGA Éditions/Université Grenoble Alpes

\section{Édition imprimée}

Date de publication : 1 mars 2001

Pagination : 175-181

ISBN : 978-2-84310-198-4

ISSN : $1240-1439$

Référence électronique

Marie-Claude Tucker, « Sir George Mackenzie of Rosehaugh, Procureur du Roi, défenseur de la sorcière Maevia en 1672 ", Études écossaises [En ligne], 7 | 2001, mis en ligne le 29 mars 2018, consulté le 08 septembre 2020. URL : http://journals.openedition.org/etudesecossaises/3469 ; DOI : https://doi.org/10.4000/etudesecossaises.3469 


\section{Sir George Mackenzie of Rosehaugh, Procureur du Roi, défenseur de la sorcière Maevia en 1672}

MARIE-Claude TUCKer Université de Clermont-Ferrand 2.

1. Reprinted in D. Webster (ed.), Collections of Rare and Curious Tracts on Witchcraft, Edinburgh, 1820.

2. Enemies of God, The Witch-hunt in Scotland, London, 1981, p. 60 .
Le tout premier pamphlet traitant des sorcières écossaises intitulé Newes from Scotland $^{1}$ fut publié à Londres en 1591. Il précédait de six années l'ouvrage du roi Jacques VI Daemonologie, publié à Édimbourg au milieu de l'une des plus sévères chasses aux sorcières. Au début du XVII ${ }^{\mathrm{e}}$ siècle, ne parut qu'un tract ayant trait à la persécution de quatre sorcières d'Irvine accusées d'avoir soulevé des tempêtes en 1618. C'est le seul exemple écossais de cette période de ce genre de tract qui expose les procès et les aveux de sorcières. Le tract n'est pas daté et il se peut d'ailleurs qu'il ne soit pas contemporain. Il faudra attendre la fin du siècle, quand la grande chasse aux sorcières sera terminée, pour disposer de documents importants. Il s'agit - et il en sera question ici - des écrits de Sir George Mackenzie of Rosehaugh, Procureur du Roi: Pleadings in some Remarkable Cases before the Supreme Courts of Scotland since the year 1661. To which the Decisions are subjoined, Edinburgh, (1672) et Laws and Customs of Scotland in Matters Criminal (1678). Ces ouvrages coïncident avec le déclin des persécutions et des arrestations de sorcières. Selon des études approfondies en la matière, il n'y eut pas de chasse aux sorcières longue et continue en Écosse mais plutôt des périodes d'intense persécution. Christina Larner ${ }^{2}$ en dénombre cinq: en 1590-1591 puis 1597, toutes les deux liées à Jacques VI, en 1629-1630, coïncidant avec la peur des sorcières sur le continent, en 1649 au moment de l'apogée de la politique du Covenant, et enfin dès 1661-1662 à l'époque de la Restauration. Pendant les années 1590-1591, il y eut d'importants procès pour trahison par sorcellerie au cours desquels il apparaît qu'environ 300 sorcières s'étaient rassemblées pour trahir le roi. Elles avaient - semblait-il - essayé d'attenter à la vie du 
3. In C. Larner, ibid., p. 6365 ; C.L. Ewen, Witch Hunting and Witch Trials, London, 1929, p. 112. En Angleterre où la population était quatre fois plus importante, le nombre de sorcières exécutées ne dépasse pas 500. A. Macfarlane, Witchcraft in Tudor and Stuart England, London, 1970, p. 62, estime ce chiffre à 300 . T.C. Smout, A History of the Scottish People 1560-1830, London, 1985, p. 184-185. Tous ces chiffres sont cependant à prendre avec grande précaution.

4. C. Larner, ibid., p. 177178. roi en soulevant des tempêtes sur la mer au moment où il rentrait du Danemark avec sa jeune épouse. Devenir ainsi la cible principale des sorcières faisait partie d'une image mythique que le roi s'appropria pour magnifier, entre autres, sa personnalité, car il n'était pas question pour lui de voir son image ternie. C'est cette inquiétude sans doute qui le poussa à mener ses recherches et à publier finalement Daemonologie en 1597.

Bien que le nombre des sorcières jugées et exécutées en Écosse nous laisse pantois aujourd'hui - entre 1000 et 1500 pour une population d'à peine un million d'habitants ${ }^{3}$ - notre attention ici ne portera que sur une seule sorcière: Maevia, dont le procès est relaté par Mackenzie lui-même dans ses Remarkable Cases. L'originalité de ces écrits réside dans le fait que Mackenzie prend la défense des sorcières pour leur éviter le bûcher et expose ses méthodes et arguments. Ses Lawes and Customs furent en réalité le premier commentaire complet d'un point de vue juridique du Witchcraft Act de 1563 qui, rappelonsle, imposait la peine de mort aux sorcières. Cette loi, qui datait en fait d'une période antérieure à la chasse aux sorcières, laissait très sceptiques car directement dirigée contre la superstition. L'acte s'étendait également à ceux qui consultaient les sorcières, bien qu'en pratique, peu de gens fussent arrêtés, semble-t-il, pour cette raison ${ }^{4}$. L'autre autorité à laquelle les hommes de loi - qu'ils fussent épiscopaliens ou presbytériens se référaient, était le Droit canon de l'Église catholique romaine. Il n'y avait pas là d'anachronisme car c'était le droit. Les hommes de loi écossais appuyaient l'Eglise dans son interprétation littérale du texte mosaïque: «thou shalt not suffer a witch to live». Il ne fait aucun doute non plus que les intellectuels écossais qui voyagaient en Europe, en particulier pour leurs études de droit, prenaient très au sérieux l'attitude sévère des autorités étrangères vis-à-vis de la menace que représentaient les sorcières.

La persécution séculière en matière de sorcellerie en Écosse se présentait de trois façons. La première avait lieu devant la cour centrale, à l'initiative le plus souvent du procureur du roi, devant les juges du roi. La deuxième avait lieu devant la circonscription judiciaire, présidée par un juge de cour centrale. La troisième se faisait par une commission de justice; un mandat d'arrêt, émis soit par le parlement soit par le conseil privé, permettait aux membres de l'élite locale, anciens et magistrats, de poursuivre et d'exécuter les sorcières. Il est à noter que ces 
derniers venaient en première position pour demander et obtenir poursuite et condamnation.

George Mackenzie, quant à lui, agit tout autrement. Originaire de Dundee, Mackenzie avait étudié le grec et la philosophie aux Universités de St Andrews et d'Aberdeen, et il n'avait pas seize ans lorsqu'il quitta l'Écosse pour la France. Pendant deux années, il devait poursuivre brillamment ses études de droit (civil et canon) à la Faculté de droit de l'Université de Bourges, qu'il quitta à la fin de l'année $1658^{5}$. Puis il retourna immédiatement en Écosse. En 1659, il fut appelé au barreau d'Édimbourg où il se fit rapidement une place exceptionnelle.

En 1661, remarqué par la hardiesse qu'il afficha au procès du marquis d'Argyll ${ }^{6}$ il fut rapidement nommé juge-adjoint, à une époque où il était courant de plaider les procès pour sorcellerie. Dès 1664, il prit une part active au développement de la Faculty of Advocates, souvent nommé à ses comités et bientôt élu doyen de cet établissement. En 1666, il fut fait chevalier, puis entama une carrière politique et entra au parlement en tant que représentant du comté de Ross. C'est donc au milieu d'une période bien remplie qu'il ne tarda pas à publier ses Pleadings on some Remarkable Cases. Dans cet ouvrage, le chapitre XVI consacré à la sorcellerie est intitulé Pleading : For Maevia, accused of witchcraft (p. 185-197).

Dans sa «Defence of a Maevia» Mackenzie relate en détail comment il défendit une femme accusée de sorcellerie. Il ne donne aucun détail personnel sur la vie de cette femme ni sur ses origines. Peut-être Maevia est-elle un résumé de plusieurs cas défendus par l'auteur. Il est à noter que Mackenzie gardera la même discrétion dans son ouvrage Lawes and Customs lorsqu'il mentionnera à titre d'exemples d'autres sorcières dont il ne livrera que le nom complet: Margaret Hutchinson,Janet Cock, Margaret Lawder ou Margaret Wallace. Maevia était doublement accusée d'une part de se transformer en colombe pour s'envoler avec deux autres sorcières vers leur lieu de réunion, et d'autre part d'avoir rendu malade une personne puis de faire disparaître la maladie à l'aide d'un charme. Mackenzie étaya

5. M-C Bellot-Tucker, Maîtres et étudiants écossais à la faculté de droit de l'Université de Bourges aux XVIe $\mathbb{E}$ $X V T^{T}$ siècles, thèse de $3^{\mathrm{e}}$ cycle, Clermont-Ferrand, Champion, 1997, à paraître. 6. $A P S$, vol. VII, p. 29. 7. Remarkable Cases, p. 185. en premier sa défense par un argument théologique afin de prouver que la sorcellerie existait bel et bien et de couper court à toute tentative éventuelle de discréditer sa défense en le taxant d'athéisme:

Yet I cannot think that our Saviour who came to dispossesse the devil..., would yet suffer him to reign like a Soveraign, as our fabulous representations would now persuade us ${ }^{7}$. 
A la suite de quoi, Mackenzie s'attaque au maléfice et, partant d'un constat scientifique, recommande la prudence car, dit-il, nous sommes ignorants des voies et faits de la nature:

As to the imposing or taking off diseases by Charmes, I conceive it is undenyable that there are many diseases whereof the Cures, as well as the Causes, are unknown to us: Nature is very subtile in its operations, and we are very ignorant in our inquiries; from the conjonction of which two arise the many errors and mistakes we committ in our reflections upon the productions of nature: to differ then from one another because of these errors is sufferable though to be regretted; but to kill one another because we cannot comprehend the reason of what each other do, is the effect of a terrible distraction; and if this were allowed the most Learned should still be in greatest danger, because they do oftimes find mysteries which astonish the ignorant; and this should give occasion to the Learned to forbear deep searches into natural mysteries, lest they should lose their life in gaining knowledge, and to persecute one another, for every Physician or Mathematician who is emulous of another, but cannot comprehend what his rival doth, would immediately make him pass for a Wizard ${ }^{8}$.

Gette déclaration à caractère scientifique précède la discussion suivante: une simple menace d'une prétendue sorcière, menace suivie d'une quelconque calamité pouvait-elle être considérée comme preuve de sorcellerie? Pour Mackenzie, la menace doit être spécifique et la calamité précise: il cite des juristes en droit canon quand il ajoute que «no malefice alone can be sufficient ground to condemn a witch, except that she either confess, or that it be proven by two famous Witnesses, that she used means that might have produc'd that effect ${ }^{9}$.

Puis il termine de ces mots: «if it were otherwise, Judges might condemn upon guessing or malice, and so more would be in danger to die by injustice than by witchcraft ${ }^{10}$.

La double charge de maléfice et de sorcellerie avait été retenue contre Maevia dans les circonstances suivantes: Maevia s'était querellée avec sa voisine qui avait alors connu une «distraction" dont Maevia l'avait guérie par la suite en lui appliquant une feuille de plantain ${ }^{11}$ sur la tempe gauche et en lui attachant au poignet un papier sur lequel était inscrit le nom

8. Ibid., p. 186. 9. Ibid., p. 188

10. Ibid.

11. Plante herbacée très commune, aux propriétés cicatrisantes et aseptisantes bien connues. de Jésus. Pour Mackenzie, sa cliente et la victime s'étaient tout simplement réconciliées et la «distraction» était intervenue en premier lieu sous l'effet de la querelle. En ce qui concernait l'acte de sorcellerie, le Procureur du Roi remarque que «there is nothing so cold as a Plantane Leaf and so it might have been 
very fit for curing a distraction, which is the most malignant and burning of all feverish distempers ${ }^{12}$.

Quant au papier portant le nom de Jésus, Mackenzie sentait bien qu'il abordait là un terrain dangereux, mais tout en concédant que l'emploi du nom de Jésus était impropre, il affirma que «to burn a poor ignorant woman, who knew not that to be evil which she used, were to make ignorance become Witchcraft and ourselves more criminal than the person we would condemn ${ }^{13}$.

De surcroît, il reprend le précepte bien connu qui va à l'encontre de toute croyance, selon lequel une sorcière ne peut à la fois infliger et guérir une maladie: «for it seems the Devil thinks that it were too much to bestow such favours upon one of his favourites $"{ }^{14}$.

Quant à l'idée que sa cliente pût voler comme une colombe avec deux autres sorcières pour se rendre à leur lieu de réunion, Mackenzie se prononce fermement: cette idée est absurde et une femme ne peut se transformer en colombe. S'appuyant sur le canon episcopi ${ }^{15}$ et saint Augustin, il conclut que toute confession émanant d'une sorcière qui avoue avoir été transformée en animal quelconque «is but an illusion of the fancy wroght by the Devil upon their melancholy brains whilst they sleep» ${ }^{16}$. Et d'ajouter: «and were it not a horrid thing to condemn innocent persons upon meer dreams ».

Enfin, Mackenzie précise que même si la femme était une sorcière, le droit civil ne punissait pas toujours tout ce que les hommes d'Église condamnaient. Parfois, le droit civil s'avérait plus libéral car les juristes «having so much more power than Divines should be careful how they punish ${ }^{17}$. La loi de 1563 Witchcraft Act signifiait la peine de mort pour les sorcières et aussi pour ceux et celles qui consultaient ces dernières. Mackenzie n'hésita pas à y faire allusion dans sa défense par scepticisme. Pour lui, seuls les moyens pour abuser des gens étaient condamnés, et dans le cas de sa cliente aucun moyen de cette sorte n'étant employé pour lui troubler la conscience, l'accusation «cannot be said to fall under the prohibition of the Act of Parliament ».

12. Ibid, p. 190.

13. Ibid. p. 191.

14. Ibid, p. 193.

15. Guide des évêques datant du $\mathrm{X}^{\mathrm{e}}$ siècle.

16. Remarkable Cases, p. 194.

17. Ibid. p. 196.
Dans son autre ouvrage Lawes and Customs of Scotland in Matters Criminal, le chapitre X consacré à la sorcellerie s'intitule simplement Witch-craft (p. 42-56). C'est un chapitre en 26 articles qui traitent de la sorcellerie en général, pour exemples: article 3 : Upon what presumptions Witches may be apprehended; article 5 : 
Paction with the Devil; article 7: the Devils mark; ou article 25: the punishment of this crime by the Civil Law and ours. Dans son article 4 intitulé who are judges competent thereto Mackenzie s'adresse amplement et directement aux juges qu'il admoneste quant aux conditions dans lesquelles ils ne devraient pas poursuivre les sorcières en justice et il conseille de façon implicite les avocats pour leur système de défense. Les accusées, remarque-t-il, sont plus souvent innocentes que coupables car ce sont en général de pauvres créatures ignorantes «and oft-times Women who understand not the nature of what they are accused of; and many mistake their own fears and apprehensions for Witchcraft; of which I shall give you two instances, one of a poor Weaver, who after he had confessed witchcraft, being asked how he saw the Devil, he answered, «like Flies dancing about the Candle». Another of a woman, who asked seriously, when she was accused, If a woman might be a Witch and not know it? And it is dangerous that these who are of all others the most simple should be tryed for a Crime which, of all others is most mysterious ${ }^{18}$.

Qui plus est, Mackenzie recommandait de veiller à ce que la personne qui se livrait à des aveux ne fût pas «weary of life, or opprest with melancholy», ce qui aurait pu la pousser à s'exclure de la communauté. Il poursuit avec cet exemple:

I went when I was a Justice Deput to examine some Women, who had confest judicially, and one of them who was a silly Creature, told me under Secresie, that she had not confest because she was Guilty, but being a poor Creature who wrought for her meat, and being defamed for a Witch she knew she would starve, for no person thereafter would either give her Meat or Lodging, and that all men would Beat her, and hound Dogs at her, and that therefore she desired to be out of the world; whereupon she wept most bitterly and upon her knees call'd God to witness to what she said ${ }^{19}$.

Mackenzie n'avait de cesse de mettre en garde contre les croyances et les pratiques de la Kirk et des Cours de Session face aux cas individuels. Il était le meilleur observateur en la matière et s'opposait aux théories qui pour lui s'avéraient impossibles, car contre tout fait scientifique. Les sorcières ne pouvaient traverser les murs et le Diable ne pouvait transformer une espèce en une autre. Cependant, il acceptait l'idée que le Diable avait pouvoir de manipuler les êtres humains

18. Laws and Customs, p. 45. sans toutefois leur conférer ce pouvoir. Le Diable est en mesure de soulever des tempêtes, puis de les calmer, d'infliger des mal- 
20. Ibid. p. 53 .

21. G. Larner, Witchcraft and Religion, The Politics of Popular Belief, Oxford, 1984, p. 17; Daemonologie, p. 7.

22. Action de piquer douloureusement toutes les parties du corps d'une sorcière pour la faire avouer. adies et de guérir ces mêmes maladies. Il peut même guérir des maladies «natural» «since he knows the natural causes and the origin of even these natural diseases, better than Physicians can, who are not present when diseases are contracted, and who being younger than he must have less experience ${ }^{20}$. C'est là un écho de la théorie de Jacques VI selon laquelle les pouvoirs du Diable émanent de la grande connaissance du monde qu'il a acquise et qui est largement supérieure à celle des hommes les plus malins ou les plus érudits de ce monde ${ }^{21}$.

Mackenzie ne doutait pas de l'existence des sorcières mais de la validité des moyens employés pour les juger. Il était presque impossible d'échapper vivant de l'accusation de sorcellerie jusque vers 1660 .

Au plus fort de la crise de sorcellerie au moment de la Restauration, Mackenzie défendit les sorcières qui se présentaient au tribunal au moyen des arguments pré-cités: circonstances, science, ou tout simplement incapacité des sorcières. Mais dans les années qui suivirent, il fit de son mieux pour les empêcher systématiquement de venir en procès, et si elles y venaient de les acquitter par manque de preuve. Il semble bien qu'une série d'acquittements et de renvois au cours des années 1670-1685 soit largement due à son influence ainsi qu'aux réformes auxquel il n'était pas étranger: il était devenu illégal de pratiquer le «pricking 22 » sans autorisation du conseil privé, comme de pratiquer la torture sous quelque forme que ce fût pour extorquer des aveux; quand des aveux étaient faits, les magistrats devaient s'assurer que les accusées s'exprimaient en pleine conscience. Ainsi s'avançait la période qui allait voir la fin des aveux, des arrestations et des exécutions. Il y eut de moins en moins de cas, et de moins en moins d'exécutions. Cependant, ce n'est qu'en 1736 que le Witchcraft Act fut abrogé. 